\title{
VASCULOPATHY IN SYSTEMIC SCLEROSIS
}

\author{
Iolanda Panaitiu ${ }^{1}$, Carina Mihai ${ }^{1}$, Roxana Sfrent-Cornatean ${ }^{1}$ \\ ${ }^{I}$ Department of Physiopathology and Immunology, \\ Carol Davila University of Medicine and Pharmacy, Bucharest, Romania \\ ${ }^{2}$ Department of Internal Medicine and Rheumatology, Cantacuzino Hospital, \\ Carol Davila University of Medicine and Pharmacy, Bucharest, Romania
}

\begin{abstract}
Systemic sclerosis (SSc) is a complex, heterogeneous, multi-organ disease, the etiology of which is not yet fully understood. Although fibrosis of the skin and major organs is a well-known, characteristic sign of this condition, the primary dysfunctions contributing to the disease phenotype appear to be vascular and immune in nature. The mechanisms leading to vasculopathy in SSc include dysregulation of vascular tone and endothelial dysfunction, followed by vascular remodeling, as well as abnormal angiogenesis and vasculogenesis. In addition, coagulopathy also plays a role in the disease process. Together, these factors produce a form of vasculopathy specific to SSc, characterized by vascular intimal proliferation in the macro- and micro-circulation, as well as qualitative and quantitative capillary changes.
\end{abstract}

Keywords: vasculopathy, systemic sclerosis, immunopathogenesis, biomarkers

\section{INTRODUCTION}

Systemic sclerosis (SSc) is a multi-faceted, heterogeneous disease of unknown etiology. Despite its high clinical variability, it is consistently characterized by three main elements: vascular alterations, autoimmunity and progressive fibrosis. However, there is increasing evidence that SSc pathogenesis is dominated by vascular changes, as demonstrated by numerous experimental and clinical studies (1).

Diagnosis is based mainly on the presence of clinical features like skin sclerosis; however, until recently, the requirements for classifying patients as having SSc lacked sensitivity for milder or early forms of the disease. Taking into account the multisystemic SSc clinical features, the American College of Rheumatology (ACR) together with the European League Against Rheumatism (EULAR) have recently developed highly sensitive and specific classification criteria for SSc (2). These criteria are also helpful for diagnosing the condition accurately and quite early, allowing early medical intervention, and presumably, improved prognosis.

According to the 2013 ACR/EULAR criteria (2), patients with skin thickening of the fingers extending proximal to the metacarpophalangeal joints are considered to have SSc without having to satisfy any other criteria. If this first criterion is not present, seven additional items are considered, each one having a numerical value reflecting its diagnostic importance. Patients scoring greater than or equal to 9 are classified as having SSc. A lesser extent of skin thickening of the fingers could consist of either puffy fingers (score=2) or sclerodactyly of the fingers (score $=4$ ), only the highest of these scores being used. Fingertip lesions include digital tip ulcers $($ score $=2)$ or fingertip pitting scars (score $=3$ ), again, only the highest score is used out of these two. The presence of telangiectasias ( score $=2$ ), abnormal nailfold capillaries (score $=2$ ), Raynaud's phenomenon (score $=3$ ) and pulmonary involvement, in the form of pulmonary arterial hypertension (PAH) and/or interstitial lung disease (score $=2$ ) are also considered. The final criterion is the presence of SSc-related autoantibodies (score $=3$ ), consisting of one of the following: anticentromere antibodies, anti-topoisomerase I antibodies and anti-RNA polymerase III antibodies. These criteria are not applicable to patients with skin thickening sparing the fingers (2).

The 2013 ACR/EULAR Classification Criteria showed improved sensitivity and specificity in the

Correspondence address:

Roxana Sfrent-Cornateanu, Lecturer, Department of Physiopathology and Immunology, Carol Davila University of Medicine and

Pharmacy, 37 Dionisie Lupu Street, Bucharest, Romania

E-mail: ccalexandr@yahoo.com 
validation cohort ( $91 \%$ and $92 \%$ respectively) compared to the 1980 ACR Classification Criteria, who had only $75 \%$ sensitivity and $72 \%$ specificity for identifying SSc cases in the same study (2). This brings an important improvement in clinical practice, by reducing the rate of $\mathrm{SSc}$ misdiagnosis (3) and including the sine scleroderma and limited cutaneous SSc subsets (2). Additionally, implementation of the new criteria allows an increased number of patients with SSc to participate in clinical or experimental studies $(2,4)$.

Once diagnosed, patients can be categorized as having limited cutaneous systemic sclerosis (lcSSc) or diffuse cutaneous systemic sclerosis (dcSSc) based on the extent of skin involvement (5). These groups also have distinctive autoantibodies and biomarkers and differ significantly in disease prognosis.

In patients with $1 \mathrm{cSSc}$, skin fibrosis is limited to the fingers, distal extremities and face (6). Raynaud's phenomenon (RP) generally precedes skin involvement and other disease manifestations, while severe complications such as scleroderma renal crisis and interstitial lung disease are rare (6). The autoantibody profile of these patients typically consists of centromere protein specific autoantibodies (7). Anti-Th/To antibodies and anti-U1RNP antibodies have also been observed in this milder form of SSc $(2,6,7)$. In the case of $1 \mathrm{cSSc}$, disease progression is slow with late development of PAH in some cases (6).

Patients with dcSSc present with skin fibrosis extended of the trunk and proximal extremities, in addition to that of the distal extremities and face. Also, $\mathrm{dcSSc}$ is characterized by rapid disease progression with early internal organ involvement (6). This more severe form of SSc has been associated with several autoantibody profiles, including anti-topoisomerase I, anti-RNA-polymerase III and anti-U3RNP specific autoantibodies (7).

Not all patients can be categorized into these two disease subsets, and the situation can change over time. There are additional forms such as SSc sine scleroderma, which presents no skin involvement at all, and SSc-overlap syndromes, in which another connective tissue disease is present concurrently with SSc (5).

This paper focuses on the vascular events contributing to this condition.

\section{VASCULAR PATHOPHYSIOLOGY IN SSC}

Vascular involvement in SSc is mainly characterized by intimal proliferation in skin, pulmonary, coronary and renal arteries, in the absence of inflammation (8). In addition, dysregulation of vascular tone, expressed in over $90 \%$ of patients as RP, plays an important role in SSc pathophysiology. The crucial element in SSc vasculopathy appears to be endothelial damage. There is evidence of endothelial cell damage preceding fibrosis, especially involving the arterioles: noticeable changes include the disappearance of membrane-bound vesicles, vacuolization of endothelial cell cytoplasm and gaps between endothelial cells (1). A decreased number of capillaries and abnormal capillary structure, also characteristic for SSc, are the result of defective vasculogenesis and angiogenesis $(5,8)$.

These vascular abnormalities apply especially to the microvasculature, and only in a smaller percentage of patients also to the larger vessels (such as the ulnar artery), resulting in various symptoms depending on which vessels are affected $(1,8)$. The most frequent vascular complication of SSc is the occurrence of digital ulcerations, in up to about $50 \%$ of patients through their entire lifetime (1). Moreover, digital ulcers were shown to be an important prognostic factor for the evolution of SSc, their presence being associated with higher mortality (9). SSc may also lead to more severe vascular complications, including PAH and scleroderma renal crisis (SRC). Approximately $15 \%$ of the patients with SSc develop PAH, which tends to occur more in $\mathrm{lcSSc}(5)$. Renal crises are less common, occurring in only $5 \%$ of patients, but are significant due to their much poorer prognosis; even with treatment, about $50 \%$ of patients progress to end-stage renal failure or die (5).

Matucci and coworkers consider that vasculopathy is the initial event in the natural course of SSc, RP being for the majority of patients the very first symptom (1). The triggers for SSc-vasculopathy are unknown, but the pathological processes include dysregulation of vascular tone and endothelial dysfunction, leading to endothelial injury. Furthermore, vascular wall remodeling resulting from this endothelial damage, abnormal angiogenesis and vasculogenesis, dysregulation of coagulation and fibrinolysis, as well as enhanced platelet aggregation in response to certain triggers are also present in SSc $(1,8)$.

\section{Dysregulation of vascular tone}

Dysregulation of vascular tone in SSc may be attributed to an imbalance between vasoconstricting and vasodilating factors $(1,8)$. High levels of endo- 
thelin-1 (ET-1), a potent vasoconstrictor, have been found in the blood vessels, lungs, kidneys and skin of SSc patients, in both early and late stages of the disease (10). ET-1 is involved in endothelial dysfunction and contributes to decreased nitric oxide (NO) levels (10). Concurrently, reduced endothelial NO synthetase expression in SSc patients also leads to a decrease in NO release and deficient vasodilation capability (11). In addition to its role as a vasodilator, Berk and his colleagues (12) demonstrated that NO has the potential to counter certain vascular alterations present in $\mathrm{SSc}$, through various mechanisms such as decreasing cytokine-induced endothelial activation and monocyte adhesion. They also found it to inhibit the release of interleukin (IL) 6 and IL-8, and prevent vascular smooth muscle cell proliferation (12). On the other hand, decreased levels of NO result in altered vascular tone and increased susceptibility of endothelial cells to oxidative damage (12). Thus, NO deficit presents an important pathogenic mechanism for arteriolar intimal proliferation and dysregulation of vascular tone (8).
The substantial body of evidence implicating the above factors as mechanisms of SSc-vasculopathy has led to the use of several pharmacological agents targeting vascular tone. Endothelin antagonists, such as bosentan and ambrisentan, have been shown to improve PAH severity and bosentan plays also a role in the prevention of digital ulcers. Another group of drugs, phosphodiesterase inhibitors, including sildenafil and tadalafil, stimulate the NO-mediated vasodilation pathway; these have also been demonstrated as being beneficial for PAH and RP (1).

\section{Endothelial injury}

The exact trigger of endothelial injury in SSc is unknown, but several mechanisms have been considered: infections with viral agents, particularly human cytomegalovirus (hCMV), abnormal cellularmediated and humoral immune responses, including antibody-dependent cellular cytotoxicity (ADCC) and anti-endothelial cell antibodies; additionally, ischemia/reperfusion injury also seems to contribute (see Fig. 1) (1,8).

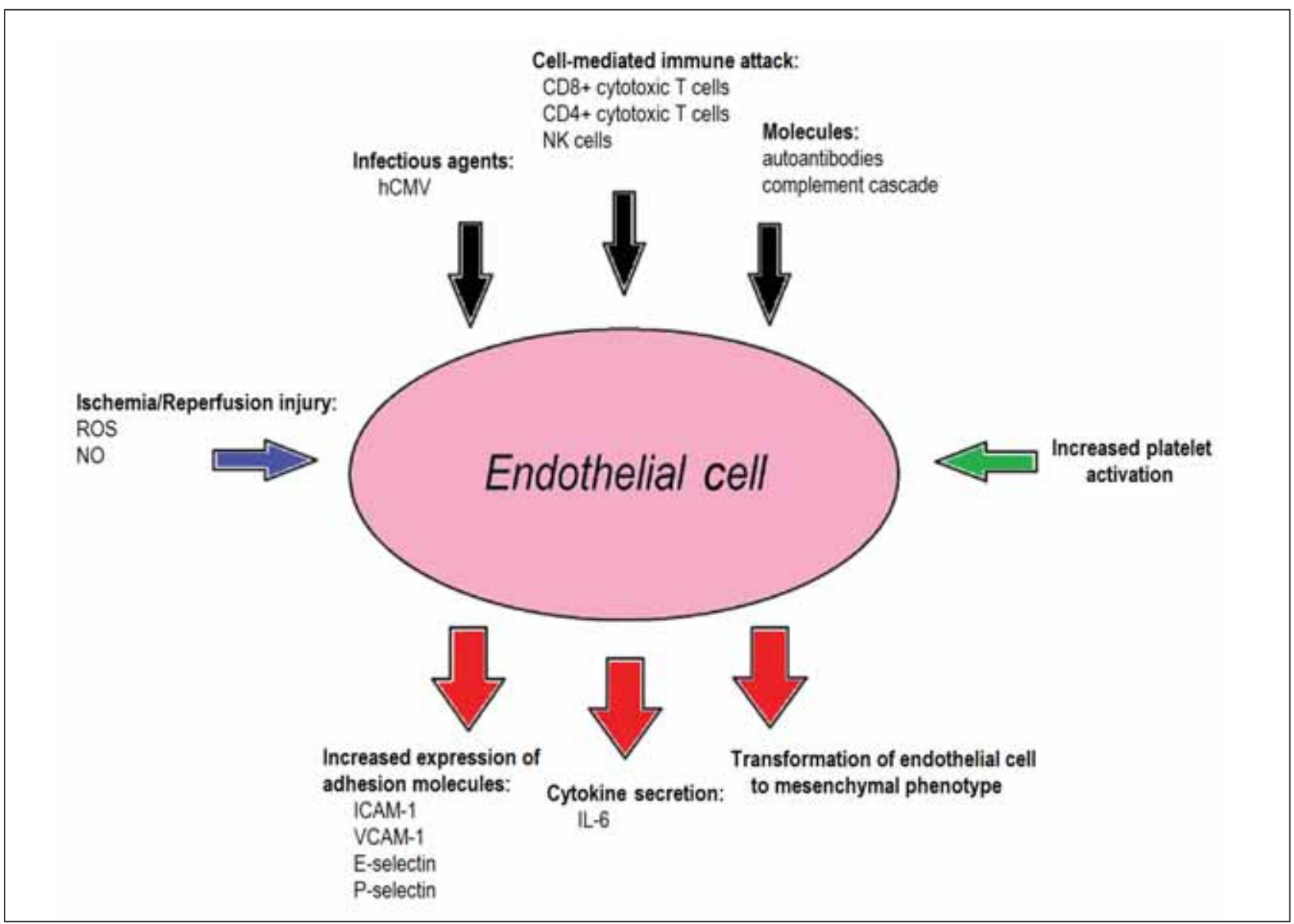

FIGURE 1. Mechanisms of endothelial dysfunction (1,8). Reactive oxygen species (ROS), nitric oxide (NO), human cytomegalovirus (hCMV), natural killer cells (NK cells), intercellular adhesion molecule 1 (ICAM-1), vascular adhesion molecule 1 (VCAM-1), interleukin 6 (IL-6). 
Kahaleh and colleagues (13) state that increased levels of hCMV antibodies have been observed in patients with SSc and that there is an association between these antibodies and vascular intimal proliferation and vasculopathy. Furthermore, the target of these antibodies shows homology with a microvasculature endothelial cell (MVEC) surface protein named tetraspan novel antigen-2 (13). These antibodies have been shown to induce MVEC apoptosis (8).

MVECs were found to be destroyed by several immune mechanisms, both cell-mediated and humoral in nature $(13,15)$. One of the proposed cellmediated mechanisms of MVEC destruction involves cells which use the granzyme-perforin system, such as cytotoxic (CD8+) T cells, natural killer cells and lymphocyte-activated killer cells, which were demonstrated to be involved in ADCC on MVECs (13). Moreover, there is also a subset of cytotoxic $(\mathrm{CD} 4+) \mathrm{T}$ cells that can induce MVEC apoptosis through the Fas-related pathway (13). Cell-mediated cytotoxicity towards MVECs could be perpetuated by the phagocytosis of apoptotic cell fragments by immature dendritic cells and macrophages, resulting in antigen presentation to $\mathrm{CD} 8+\mathrm{T}$ cells (16). Regarding humoral immune mechanisms, these same fragments may also serve to activate the alternative complement pathway, as well as promote coagulation, contributing to vasculopathy (8). Finally, anti-endothelial antibodies have been demonstrated to induce MVEC apoptosis in vitro (16).

Ischemia/reperfusion injury, mostly associated with RP, is accompanied by up-regulation of cellular adhesion molecules (CAMs). Increased CAMs allow platelet and neutrophil attachment, leading to MVEC injury due to superoxide radical production and subsequent decrease in vasodilating substances such as NO and prostacyclin $(8,13)$. Exposed subendothelial tissue, resulting from endothelial injury, forms a site for platelet aggregation, finally leading to intravascular thrombus formation (17).

\section{Defective angiogenesis}

Any structural defects at the microvascular level would normally be repaired by either angiogenesis (which is defined as compensatory growth of new vessels from existing ones) or vasculogenesis (de novo formation of new blood vessels involving stem or progenitor cells) (1). There is evidence of impaired angiogenic response, resulting in the loss of capillaries and small blood vessels in patients with
SSc $(1,18)$. This occurs despite an apparent increase in various pro-angiogenic factors including growth factors, cytokines, chemokines, vasoactive factors, cellular adhesion molecules, such as E and P selectins, and other molecules $(8,18,19)$ (see Table 1). One of the most important and well studied pro-angiogenic factors is the vascular endothelial growth factor (VEGF). Chronic overexpression of VEGF along with impaired VEGF receptor signaling are thought to be the cause of the large, irregularly shaped capillaries that are characteristic of SSc $(1,18)$.

TABLE 1. Mediators of angiogenesis $(1,8,18,19)$

\begin{tabular}{|c|c|c|}
\hline & $\begin{array}{l}\text { Pro-angiogenic } \\
\text { mediators }\end{array}$ & $\begin{array}{l}\text { Anti-angiogenic } \\
\text { mediators }\end{array}$ \\
\hline $\begin{array}{l}\text { GROWTH } \\
\text { FACTORS }\end{array}$ & $\begin{array}{l}\text { VEGF, FGF- } 2 \text {, PDGF, } \\
\text { TGF- } \alpha \text {, TGF- } \beta \text {, HGF, } \\
\text { IGF, G-CSF, BFGF, } \\
\text { GM-CSF, PIGF, } \\
\text { Angiopoietin-1, }\end{array}$ & Angiopoietin-2 \\
\hline CYTOKINES & $\begin{array}{l}\text { TNF- } \alpha \text {, IL-6, } \\
\text { Erythropoietin }\end{array}$ & $\begin{array}{l}\text { IFN- } \alpha, \text { IFN- } \beta, \text { IL-12, } \\
\text { IL-4 }\end{array}$ \\
\hline CHEMOKINES & $\begin{array}{l}\text { MCP-1, IL-8 (CXCL8), } \\
\text { CX3-CL1, CXCL12 }\end{array}$ & CXCL10, CXCL9 \\
\hline $\begin{array}{l}\text { VASOACTIVE } \\
\text { FACTORS }\end{array}$ & ET-1 & \\
\hline $\begin{array}{l}\text { OTHER } \\
\text { MOLECULES }\end{array}$ & \begin{tabular}{|l|} 
Tissue Kallicrein, \\
Kallicrein 9,11,12, \\
Metalloproteinases \\
(MMP-9, pro-MMP-1), \\
UPAR
\end{tabular} & $\begin{array}{l}\text { Angiostatin, } \\
\text { Endostatin, } \\
\text { Thrombospondin-1,2, } \\
\text { PAF, Tissue } \\
\text { inhibitors of } \\
\text { metalloproteinases, } \\
\text { Pentraxin 3 }\end{array}$ \\
\hline
\end{tabular}

Vascular endothelial growth factor (VEGF), fibroblast growth factor-2 (FGF-2), platelet derived growth factor (PDGF), tumor growth factor- $\alpha$, $\beta$ (TGF- $\alpha, \beta)$, hepatocyte growth factor (HGF), insulin-like growth factor, (IGF), basic fibroblast growth factor (BFGF), granulocyte-colony stimulatory factor (G-CSF), granulocyte-macrophage colony stimulatory factor (GM-CSF), placental growth factor (PIGF), tumor necrosis factor- $\alpha$ (TNF- $\alpha$ ), interleukin-6 (IL-6), interleukin-4 (IL-4), interleukin-12 (IL-12), monocyte chemoattractant protein-1(MCP-1), interleukin-8 (IL-8), interferon $\alpha, \beta$ (IFN $\alpha, \beta)$, urokinase plasminogen activator receptor (UPAR).

The rarefaction of small blood vessels is likely due to an imbalance between pro-angiogenic and angiostatic factors, with an even greater upregulation of the latter. The anti-angiogenic factors that are increased in SSc are listed in Table $1(8,19)$.

Some of these pro-angiogenic molecules are also important activators of vascular smooth muscle cells, pericytes and stromal fibroblasts, providing a link between the vascular and fibrotic events in SSc (20). Pericytes, which play a crucial role in angiogenesis by stabilizing the developing vessel and allowing it to mature, show increased expression of regulator of G-protein signalling (RGS-5), a negatively regulating angiogenic factor $(8,18)$. Further- 
more, pericytes of SSc patients have a tendency towards increased proliferation, leading to increased vascular wall thickness and decreased lumen size (21). Also, increased platelet activity could promote defective angiogenesis by contributing to the imbalance between the stimulators and inhibitors of this process (8).

\section{Defective vasculogenesis}

Circulating endothelial progenitor cells (EPCs) seem to be key players in SSc vasculopathy, with respect to vasculogenesis; these cells would normally be able to initiate and restore the vascular supply to ischemic tissue (1). However, there have been conflicting experimental results in terms of the role and presence of EPCs in SSc: some studies have reported increased levels, while others have observed decreased EPC levels $(8,18)$. Different experimental protocols, medications the patients were taking and the severity of the disease when the samples were taken are only a few of the many factors that could explain this discrepancy (18).

Kuwana et al. (2004) found that SSc patients with pitting scars and active fingertip ulcers had the lowest levels of EPCs (22). Furthermore, the EPCs showed impaired maturation capacity; early outgrowth EPCs of these patients showed a lack of mature endothelium markers, such as VE-cadherin, CD146 and von Willebrand Factor (22). On the other hand, early SSc with high disease activity was associated with increased levels of EPCs, which showed a positive correlation with peripheral vascular manifestations (23). With disease progression, some studies noted a drop in EPC levels, which corresponded to endothelial dysfunction, capillary loss and the development of severe cardiac involvement and PAH (23).

There seems to be a trend of increased EPC levels at the beginning of the disease, perhaps due to EPC mobilization to hypoxic tissues, while these levels drop significantly in late-stage SSc, possibly due to bone marrow depletion $(18,23)$. Other theories for decreased levels of EPCs include an impaired bone marrow microenvironment for endothelial stem cells (24) and SSc patient serum toxicity for EPCs due to IgG antibody involvement (25).

Avouac et al. (2008) demonstrated that SSc lateoutgrowth EPCs failed to up-regulate VEGFR-1 in hypoxic conditions; consequently, VEGF might preferentially interact with VEGFR-2, leading to chronic stimulation by VEGF, resulting in abnormal vessel shape and functionality (26).

\section{Endothelial transformation to a mesenchymal phenotype}

Endothelial transformation to a mesenchymal phenotype, expressing $\alpha$-smooth muscle actin, vimentin and collagen, can be detected in SSc patients (17). This transformation may be due to local growth factors and cytokines, especially TGF $\beta$ (27). Upregulation of the transcriptional repressor snail-1 (which forms a complex with Smad3/Smad4) induces transcriptional events that could lead to this functional modification (28). Other factors that seem to be involved include PDGF, VEGF, insulin-derived GF, CTGF, ET-1 and several miRNAs (8). Decreased values of miRNA-21, miRNA-155 and miRNA-126 have especially been associated with vascular disease (29).

\section{Coagulopathy}

The vascular alterations in SSc include coagulopathy in the form of microvascular thrombosis and enhanced fibrin deposition (8). Impaired fibrinolysis is $\mathrm{SSc}$ may be a consequence of defective tissue plasminogen activator (tPA) release (8) or increased levels of tPA inhibitor (PAI) (30). Increased coagulation activation, as well as elevated levels of fibrinogen and vWF have been found in SSc patients (31). Excessive inactivation of plasmin by $\alpha 2$-antiplasmin is associated with fibrosis and has been observed in SSc patients (32). $\alpha 2$-antiplasmin increases TGF $\beta$ levels by activating the phospholipase A2 pathway (32).

\section{Platelet abnormalities}

Platelets are important players in SSc pathogenesis, because their contribution to the development of this disease seems to involve many different pathways including defective angiogenesis: their granule content includes some pro-angiogenic factors, such as VEGF, PDGF, and TGF and also, some anti-angiogenic factors, including endostatin and thrombospondin-1 (18).

Chronic platelet activation and product release (many of which are inflammatory mediators) contribute to SSc pathology (33). Platelet derived inflammatory factors include nitric oxide, serotonin, thromboxane A2, prostaglandin-D2, prostaglandinE2, prostaglandin-F2, 12-hydroxy-eicosatetraeonic 
acid, $\beta$-thromboglobulin, neutrophil-activating peptide-2, platelet factor-4, platelet activating factor, adenosine, histamine, P-selectin, CD40 ligand, dinucleoside polyphosphatases and 2-arachidonyl glyceride (33).

Enhanced platelet aggregation in response to collagen, adenosine diphosphates, 5-hydroxytryptamine and endothelin-1, is also present in SSc $(33,34)$. This is due to the overexpression of phosphatidylinositol-3, a collagen receptor, as well as increased nitrosylation and protein B kinase activity $(33,35)$. Platelets also store numerous fibrogenic mediators, including TGF $\beta 1$, TGF $\beta 2$, PDGF A,B,C,D, adenosine, CTGF and IGF, contributing to the chronic tissue fibrosis (36).

\section{BIOLOGICAL MARKERS INDICATING VAS- CULAR INVOLVEMENT IN SSC}

Several biological markers have been suggested as indicators of endothelial injury in SSc, including circulating levels of von Willebrand factor (vWF), vascular endothelial growth factor (VEGF), endothelin-1 (ET-1), thrombomodulin, thrombospondin, brain natriuretic peptide (BNP), endostatin, plasminogen activator, prostacyclin, thromboxane and circulating metabolites of $\mathrm{NO}(7,37)$. VEGF is elevated in SSc patients; one study found a positive correlation between VEGF levels and systemic organ involvement (38). Others postulate that chronic VEGF upregulation (39) or impaired VEGF signaling (26) could be a cause of vascular abnormalities. Elevated vWF plasma levels have been linked with increased disease severity and early pulmonary involvement (7).

Circulating viable and dead endothelial cells (40) are important markers of endothelial damage, in addition to microparticles (41), which are small vesicles released into the circulation by distressed endothelial cells. These endothelial microparticles have been reported to be elevated in SSc and could constitute useful markers for disease progression and vascular involvement. Plasma levels of CD40 ligand are also elevated in SSc and seem to be associated with the presence of PAH and digital ulcerations (42).

Endothelial injury stimulates the expression of cellular adhesion molecules (CAMs), but the resulting recruitment of inflammatory cells may further damage the endothelium (7,37). Elevated levels of circulating ICAM-1 were associated with dcSSc, rapid disease progression and the presence of digital ulcers (43). Circulating levels of ICAM-1, P-selectin, vascular cell adhesion protein (VCAM)-1 and E-selectin were all demonstrated to positively correlate with disease activity (7).

Endothelial cells in the skin of patients with early $\mathrm{dcSSc}$ have been observed to express higher levels of IL-6 (44). Some studies have demonstrated a positive correlation between IL-6 and extent of skin thickening $(44,45)$; higher levels were indicative of poor prognosis. Serum levels of IL-6 were demonstrated to be an independent predictor of the diffusion capacity for carbon monoxide decline in SSc interstitial lung disease (46).

The increased expression of angiostatic molecules in SSc patients rivals that of pro-angiogenic factors. Angiostatin, endoglin, endostatin thrombospondin and VEGF receptor are all elevated and have been suggested as possible markers for SSc vasculopathy (7). A study by Hummers et al. (2009) found elevated plasma levels of endostatin that correlated positively with right ventricular systolic pressure, which is indicative of PAH (47). On the other hand, soluble endoglin levels were increased in SSc patients with skin ulcerations and in those positive for anticentromere antibodies (48). Elevated levels of soluble endoglin are more likely found in lcSSc patients and are indicative of PAH prognosis (49).

TABLE 2. Biomarkers indicating vasculopathy in SSC $(7,37-44,46)$

\begin{tabular}{|l|l|}
\hline Growth factors & VEGF* \\
\hline Cytokines & IL-6 \\
\hline $\begin{array}{l}\text { Adhesion } \\
\text { molecules }\end{array}$ & ICAM-1*, VCAM-1*, E-selectin*, P-selectin \\
\hline $\begin{array}{l}\text { Vasoactive } \\
\text { factors }\end{array}$ & $\begin{array}{l}\text { ET-1*, prostacyclin, thromboxane, NO } \\
\text { metabolites }\end{array}$ \\
\hline $\begin{array}{l}\text { Angiostatic } \\
\text { factors }\end{array}$ & $\begin{array}{l}\text { Angiostatin, endoglin, endostatin, } \\
\text { thrombospondin }\end{array}$ \\
\hline Other & $\begin{array}{l}\text { VWF* } \\
\text { CD40 ligand } \\
\text { thrombomodulin } \\
\text { plasminogen activator } \\
\text { BNP } \\
\text { circulating endothelial cells } \\
\text { endothelial cell fragments }\end{array}$ \\
\hline
\end{tabular}

Vascular endothelial growth factor (VEGF), interleukin 6 (IL-6), intercellular adhesion molecule 1 (ICAM-1), vascular cell adhesion molecule 1 (VCAM-1), endothelin 1 (ET-1), von Willebrand factor (VWF), brain natriuretic peptide (BNP).

*These molecules are well-known and established biomarkers for SSC (37).

Despite a rather long list of possible biological markers indicating vascular involvement in SSc, there are a few, widely acknowledged ones, which should always be kept in mind for assessing disease 
activity or treatment response. These include vWF, ET-1, ICAM-1, VCAM-1 and E-selectin, which signal endothelial damage, and VEGF, a potent angiogenic factor $(7,37)$.

\section{CONCLUSION}

In conclusion, $\mathrm{SSc}$ is a complex disease with numerous, intricate mechanisms that are not yet fully understood. The new ACR/EULAR classification

\section{REFERENCES}

1. Matucci-Cerinic M., Kahaleh B., Wigley F.M. Review: evidence that systemic sclerosis is a vascular disease. Arthritis Rheum. 2013; 65(8): 1953-62.

2. van den Hoogen F., Khanna D., Fransen J., et al. 2013 classification criteria for systemic sclerosis: an American College of Rheumatology/European League against Rheumatism collaborative initiative. Arthritis Rheum. 2013; 65(11): 2737-47.

3. Dobrota R., Soare A., Gherghe A.M., et al. Performance of the 2013 ACR/EULAR Classification Criteria for Systemic Sclerosis in a Single Center Setting, Clin Exp Rheumatol. 2014; 32(2), Suppl.81, S-7.

4. Sáez-Comet L., et al. Applying the ACR/EULAR Systemic Sclerosis Classification Criteria to the Spanish Scleroderma Registry Cohort. J Rheumatol. 2015; 42(12): 2327-31.

5. Allanore Y., Simms R., Distler O., et al. Systemic sclerosis. Nature Reviews Disease Primers. 2015; 1:1-21.

6. LeRoy E.C., et al. Scleroderma (systemic sclerosis): classification, subsets and pathogenesis. J. Rheumatol. 1988; 15: 202-205.

7. Hasegawa M. Biomarkers in systemic sclerosis: Their potential to predict clinical courses. Journal of Dermatology. 2016; 43: 29-38.

8. Pattanaik D., Brown M., Postlethwaite B.C., Postlethwaite A.E. Pathogenesis of systemic sclerosis. Front. Immunol. 2015; 6: 272.

9. Mihai C., Landewé R., van der Heijde D., et al. Digital ulcers predict a worse disease course in patients with systemic sclerosis. Ann Rheum Dis. 2016; 75(4): 681-6.

10. Abraham D.J., Krieg T., Distler J., et al. Overview of pathogenesis of systemic sclerosis. Rheumatology. 2009; 48(suppl 3): iii3-iii7

11. Grygiel-Górniak B., Puszczewicz M. Oxidative Damage and Antioxidative Therapy in Systemic Sclerosis. Mediators of Inflammation. 2014; 2014: 389582.

12. Berk B.C., Abe J.I., Min W., et al. Endothelial atheroprotective and anti-inflammatory mechanisms. Ann N Y Acad Sci. 2001; 947: 93-109.

13. Kahaleh B. The microvascular endothelium in scleroderma. Rheumatology. 2008; 47(Suppl 5): v14-5.

14. Ahmed S.S., Tan F.K., Arnett F.C., et al. Induction of apoptosis and fibrillin 1 expression in human dermal endothelial cells by scleroderma sera contain-ing anti-endothelial cell antibodies. Arthritis Rheum. 2006; 54(7): 2250-62.

15. Albert M.L., Jegathesan M., Darnell R.B. Dendritic cell maturation is required for the cross-tolerization of CD8+ T cells. Nat Immunol. 2001; 2(11): 1010-7.

16. Mihai C., Tervaert J.W.C. Anti-endothelial cell antibodies in systemic sclerosis. Ann Rheum Dis. 2010; 69: 2319-324.

17. Jimenez S.A. Role of endothelial to mesenchymal transition in the pathogenesis of the vascular alterations in systemic sclerosis. ISRN Rheumatol. 2013; 2013: 835948

18. Manetti M., Guiducci S., Ibba-Manneschi L., Matucci-Cerinic M. Mechanisms in the loss of capillaries in systemic sclerosis: angiogenesis versus vasculogenesis. J. Cell. Mol. 2010; 14(6A): $1241-1254$.

19. Mulligan-Kehoe M.J., Drinane M.C., Mollmark J., et al. Antiangiogenic plasma activity in patients with systemic sclerosis. Arthritis Rheum. 2007; 56: 3448-58. criteria have improved our ability to recognize and diagnose this condition, but our capacity to prevent or treat SSc remains limited. It is important to continue investigating SSc pathogenesis, especially its significant vascular involvement, as this seems to be the primary pathogenic event. Further research in this area could provide opportunities to identify additional targets for pharmacological intervention in order to stop or even reverse disease progression.

20. Distler O., Pap T., Kowal-Bielecka O., et al. Overexpression of monocyte chemoattractant protein 1 in systemic sclerosis: role of platelet-derived growth factor and effects on monocyte chemotaxis and collagen synthesis. Arthritis Rheum. 2001; 44: 2665-78.

21. Helmbold P., Fiedler E., Fischer M., Marsch W. Hyperplasia of dermal micro-vascular pericytes in scleroderma. J Cutan Pathol. 2004; 31(6): 431-40.

22. Kuwana M., Okazaki Y., Yasuoka H., et al. Defective vasculogenesis in systemic sclerosis. Lancet. 2004; 364: 603-10.

23. Nevskaya T., Bykovskaia S., Lyssuk E., et al. Circulating endothelial progenitor cells in systemic sclerosis: relation to impaired angiogenesis and cardiovascular manifestations. Clin Exp Rheumatol. 2008; 26: 421-9.

24. Cipriani P., Guiducci S., Miniati I., et al. Impairment of endothelial cell differentiation from bone marrow-derived mesenchymal stem cells: new insight into the pathogenesis of systemic sclerosis. Arthritis Rheum. 2007; 56: 1994-2004.

25. Zhu S., Evans S., Yan B., et al. Transcriptional regulation of Bim by FOXO3a and Akt mediates scleroderma serum-induced apoptosis in endothelial progenitor cells. Circulation. 2008; 118: 2156-65.

26. Avouac J., Wipff J., Goldman O., et al. Angiogenesis in systemic sclerosis: impaired expression of vascular endothelial growth factor receptor 1 in endothelial progenitor-derived cells under hypoxic conditions. Arthritis Rheum. 2008; 58(11): 3550-61.

27. Medici D., Potenta S., Kalluri R. Transforming growth factor-beta2 promotes Snail-mediated endothelial-mesenchymal transition through convergence of Smad-dependent and Smad-independent signalling. Biochem J. 2011; 437(3): 515-20.

28. Li Z., Jimenez S.A. Protein kinase Cdelta and c-Abl kinase are required for trans-forming growth factor beta induction of endothelial-mesenchymal transition in vitro. Arthritis Rheum. 2011; 63(8): 2473-83.

29. Urbich C., Kuenbacher A., Dimmeler S. Role of microRNAs in vascular diseases, inflammation, and angiogenesis. Cardiovascular Research. 2008; 79: 581-588.

30. Kessler-Becker D., Smola S., Krieg T., et al. High plasminogen activator inhibitor type 2 expression is a hallmark of scleroderma fibroblasts in vitro. Exp Dermatol. 2004; 13(11): 708-14.

31. Asano Y., Sato S. Vasculopathy in scleroderma. Semin Immunopathol. 2015; 37: 489-500.

32. Kanno Y., Kawashita E., Kokado A., et al. Alpha2-antiplasmin regulates the development of dermal fibrosis in mice by prostaglandinF (2alpha) synthesis through adipose triglyceride lipase/calcium independent phospholipase A(2). Arthritis Rheum. 2013; 65(2): 492-502.

33. Postlethwaite A.E., Chiang T.M. Platelet contributions to the pathogenesis of systemic sclerosis. Curr Opin Rheumatol. 2007; 19(6): 574-9.

34. Pattanaik D., Postlethwaite A.E. A role for lysophosphatidic acid and sphingosine 1-phosphate in the pathogenesis of systemic sclerosis. Discov Med. 2010; 10(51): 161-7.

35. Chiang T.M., Postlethwaite A.E. A cell model system to study regulation of phosphatidylinositol 3-kinase and protein kinase $B$ activity by cytokines/growth factors produced by type I collagen 
stimulated immune cells from patients with systemic sclerosis. Biochim Biophys Acta. 2007; 1770(8): 1181-6.

36. Pattanaik D., Brown M., Postlethwaite A.E. Vascular involvement in systemic sclerosis (scleroderma). J Inflamm Res. 2011; 4: $105-25$.

37. Hummers L.K. The Current State of Biomarkers in Systemic Sclerosis. Curr Rheumatol Rep. 2010; 12(1): 34-39.

38. De Santis M., Ceribelli A., Cavaciocchi F., et al. Nailfold videocapillaroscopy and serum VEGF levels in scleroderma are associated with internal organ involvement. Auto Immun Highlights. 2016; 7(1): 5 .

39. Distler O., Distler J.H., Scheid A., et al. Uncontrolled expression of vascular endothelial growth factor and its receptors leads to insufficient skin angiogenesis in patients with systemic sclerosis. Circ Res. 2004; 95(1): 109-116.

40. Del Papa N., Colombo G., Fracchiolla N., et al. Circulating endothelial cells as a marker of ongoing vascular disease in systemic sclerosis. Arthritis Rheum. 2004; 50(4): 1296-304.

41. Jung C., Drummer K., Oelzner P., et al. The association between endothelial microparticles and inflammation in patients with systemic sclerosis and Raynaud's phenomenon as detected by functional imaging. Clin Hemorheol Microcirc. 2016; 61(3): 549-57.

42. Allanore Y., Borderie D., Meune C., et al. Increased plasma soluble CD40 ligand concentrations in systemic sclerosis and association with pulmonary arterial hypertension and digital ulcers. Ann Rheum Dis. 2005; 64: 481-483.
43. Bhattacharyya S., Wei J., Varga J. Understanding fibrosis in systemic sclerosis: shifting paradigms, emerging opportunities. Nat Rev Rheumatol. 2012; 8(1): 42-54.

44. Khan K., Xu S., Nihtyanova S., et al. Clinical and pathological significance of interleukin 6 overexpression in systemic sclerosis. Ann Rheum Dis. 2012; 71(7): 1235-1242.

45. Sato S., Hasegawa M., Takehara K. Serum levels of interleukin-6 and interleukin-10 correlate with total skin thickness score in patients with systemic sclerosis. J Dermatol Sci. 2001; 27: 140-146.

46. De Lauretis A., Sestini P., Pantelidis P. et al. Serum interleukin 6 is predictive of early functional decline and mortality in interstitial lung disease associated with systemic sclerosis. J Rheumatol. 2013; 40(4): 435-446.

47. Hummers L.K., Hall A., Wigley F.M., Simons M. Abnormalities in the regulators of angiogenesis in patients with scleroderma. J Rheumatol. 2009; 36(3): 576-582.

48. Wipff J., Avouac J., Borderie D., et al. Disturbed angiogenesis in systemic sclerosis: high levels of soluble endoglin. Rheumatology (Oxford). 2008; 47(7): 972-975

49. Fujimoto M., Hasegawa M., Hamaguchi Y., et al. A clue for telangiectasis in systemic sclerosis: elevated serum soluble endoglin levels in patients with the limited cutaneous form of the disease. Dermatology. 2006; 213(2): 88-92. 